\title{
On Wireless Social Community Networks
}

\author{
Mohammad Hossein Manshaei*, Julien Freudiger*, Mark Félegyházi*, Peter Marbach ${ }^{\dagger}$, and Jean-Pierre Hubaux* \\ * Laboratory for computer Communications and Applications (LCA), EPFL, Lausanne, Switzerland \\ Email:\{hossein.manshaei, julien.freudiger, mark.felegyhazi, jean-pierre.hubaux\}@epfl.ch \\ ${ }^{\dagger}$ Department of Computer Science, University of Toronto, Canada \\ Email: marbach@cs.toronto.edu
}

\begin{abstract}
Wireless social community networks are emerging as a new alternative to provide wireless data access in urban areas. By relying on users in the network deployment, a wireless community can rapidly deploy a high-quality data access infrastructure in an inexpensive way. But, the coverage of such a network is limited by the set of access points deployed by the users. Currently, it is not clear if this paradigm can serve as a replacement of existing centralized networks operating in licensed bands (such as cellular networks) or if it should be considered as a complimentary service only. This question currently concerns many wireless network operators. In this paper, we study the dynamics of wireless social community networks using a simple analytical model. In this model, users choose their service provider based on the subscription fee and the offered coverage. We show how the evolution of social community networks depends on their initial coverage, the subscription fee, and the user preferences for coverage. We conclude that by using an efficient static or dynamic pricing strategy, the wireless social community can obtain a high coverage. Using a game-theoretic approach, we then study a case where the mobile users can choose between the services provided by a licensed band operator and those of a social community. We show that for specific distribution of user preferences, there exists a Nash equilibrium for this noncooperative game.
\end{abstract}

\section{INTRODUCTION}

Wireless networks have traditionally been deployed and operated by central authorities. The centralized management of wireless network infrastructures guarantees a high quality of service (QoS) in terms of network coverage, but at the expense of substantial deployment and maintenance costs. Having users form wireless social communities, a wireless network operator can share infrastructure costs with its customers. The WiFi technology (i.e., IEEE 802.11 devices) is a viable option: WiFi networks offer an inexpensive high-speed wireless access to users and do not necessitate expensive investments because the technology operates in an unlicensed frequency band. Thus, there is no need for the operator to make substantial initial investment to buy the spectrum license. Furthermore, the access points (AP) are inexpensive, easy to deploy and maintain. Still, the wireless social community typically has a limited coverage, which depends on the size of the network. Wireless social community networks could be at the core of the next wireless generation.

In this paper, we are concerned with the potential of wireless social communities to compete with traditional licensed band networks. We first evaluate the evolution of wireless social community networks by modeling users' payoffs as a function of the subscription fee ${ }^{1}$, as well as the operators' provided coverage. We discuss static and dynamic strategies for attracting new subscribers to improve the coverage of social community networks. To the best of our knowledge, this is the first model to address and evaluate the strategies of social community operators, taking into account the preferences of users in term of coverage and subscription fees.

Then, we discuss the competition between social community operators and traditional licensed band operators using a game-theoretic approach. We investigate the strategies of the operators in a competition and the corresponding outcomes of the game. In the hope of mutually beneficial results, we identify a Nash equilibrium in this game and discuss the possible cooperation between the operators. We believe that our paper gives an insight to understanding the evolution of wireless social communities in the presence of traditional wireless access providers.

The paper is organized as follows. In Section II, we characterize the properties of users, the licensed band operator and the social community operator. In Section III, we present the main results and contributions of this paper. In Section IV and $\mathrm{V}$, we evaluate the dynamics of these networks separately and derive the maximum payoff and the corresponding optimal number of subscribers. In Section VI, we model the competition of these two types of network operators and discuss their coexistence. Finally, in Section VII, VIII, and IX we discuss the related work, conclusions and some open questions.

\section{SySTEM MOdEL}

We consider a network service area, where $N$ users ( $N$ is very large) have the choice between the services offered by two wireless network operators. We assume that one operator deploys his own network infrastructure in a licensed band (e.g., WiMAX) to provide wireless access to users. The other operator relies on technologies operating in unlicensed bands (e.g., WiFi) and involves the wireless APs operated by the users to establish a wireless social community. Consequently, we refer to the two operators as the licensed band operator $(L B O)$ and the social community operator (SCO).

In our model, there exists a discrete sequence of time, i.e., $\{t=1,2, \cdots, \infty\}$, at which each user makes decision whether to subscribe to a given operator depending on its payoff. At

\footnotetext{
${ }^{1}$ Note that the subscription fee corresponds to the price users have to pay. Hence, we use the two terms interchangeably in the paper.
} 
the same time, each operator calculates the fraction of users that subscribed to its service $\left(0 \leq n_{i}[t] \leq 1\right)$ and computes its payoff $\left(u_{i}[t]\right.$ ), where $i \in\{\ell, s\}$ ( $\ell$ and $s$ represent LBO and SCO respectively).

Let $Q_{i} \in[0,1]$ and $P_{i}, i \in\{\ell, s\}$ be the provided coverage and subscription fee by a given network operator $\left(Q_{i}=1\right.$ means full coverage). We assume that the users evaluate the usefulness of the networks based on the provided coverage and subscription fee. The study of more sophisticated user preferences is part of our future work (as discussed in Section IX). We define the nature of the pricing strategy as follows:

Definition 1: With a static pricing strategy, an operator does not change the subscription fee $P_{i}$ at decision times $t$.

Definition 2: With a dynamic pricing strategy, an operator can change its price $P_{i}$ at decision times $t$.

Next, we characterize the payoff functions of two operators as well as the users.

\section{A. Licensed Band Operator (LBO)}

LBOs typically use a collision free protocol (e.g., WiMAX) and licensed spectrum to provide the wireless access. We suppose that the LBO has full coverage (i.e., $Q_{\ell}=1$ ). We denote the payoff of the LBO at time $t$ by $u_{\ell}[t]$. This payoff is a function of $n_{\ell}[t]$ and the cost of the LBO infrastructure $c_{\ell}$ (e.g., the cost to deploy and maintain base stations, to acquire the spectrum licenses, etc.). Hence, we define the payoff of the $\mathrm{LBO}$ at time $t$ as:

$$
u_{\ell}[t]=N \cdot n_{\ell}[t] \cdot P_{\ell}-c_{\ell} .
$$

\section{B. Social Community Operator ( $\mathrm{SCO}$ )}

Subscribers to the SCO participate in the deployment of the network in the unlicensed band (e.g., by sharing their IEEE 802.11 APs). We assume that the users pay a monthly subscription fee $P_{s}$ to the SCO to be a member of the community. This subscription fee is most likely to be substantially smaller than the LBO subscription fee $P_{\ell}$. We assume that for this price, the SCO provides the APs to the users and maintains the network infrastructure (e.g., the software that enables social community services). Thus, the SCO has a small cost $c_{s}$ for deploying the service. We assume that SCOs previously agreed with ISPs to let users share their APs. We discuss the strategic service agreements between ISPs and SCOs [2] in Section IX.

The coverage $Q_{s}$ of the social network, unlike for the LBO, depends on the number of users who subscribed to the SCO network. We assume that $Q_{s}$ is a linear function of $n_{s}[t]$, as shown in Fig. 1(a), i.e., $Q_{s}[t]=n_{s}[t]$.

We also assume that the best coverage (i.e., $Q_{s}=1$ ) is obtained if and only if all users subscribe to the community. With this coverage function, we assume a channel assignment scheme as introduced in [7], i.e., a non-interfering channel assignment obtained via local channel bargaining. Similarly to the LBO, the payoff function of the $\mathrm{SCO}$ at time $t$ is:

$$
u_{s}[t]=N \cdot n_{s}[t] \cdot P_{s}-c_{s}
$$

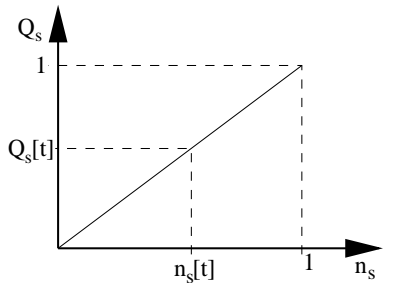

(a)

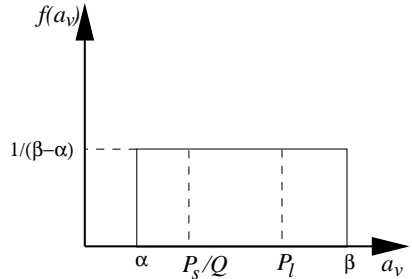

(b)
Fig. 1. System model: (a) Relation between subscribers and the social community coverage at time $t$. (b) Uniform distribution of user types.

\section{User Model}

In our model, users subscribe to a wireless network operator based on the provided coverage as well as their sensitivity to it and the subscription fee. Assume that user $v$ subscribed to operator $i \in\{\ell, s\}$. Then, we model the payoff of user $v$ as a function of the coverage provided by network operator $Q_{i}$, the subscription fee of operator $P_{i}$, and a user type parameter $a_{v}$ that characterizes its sensitivity to provided coverage. For any user $v$ the payoff under operator $i$ is:

$$
u_{v}^{i}=a_{v} \cdot Q_{i}-P_{i} .
$$

A user $v$ subscribes to an operator if its payoff using that operator is greater than zero, i.e., $u_{v}^{i}>0$. Note that we consider a payoff function $u_{v}$ that depends linearly on the available coverage. For instance, the users with low $a_{v}$ require high $Q_{i}$ to subscribe to operator $i$. A fraction of users with very small $a_{v}$ will refrain from subscribing to any operator, because they are not satisfied with the available coverage. We discuss the extension of the model to generic concave payoff functions in Section IX. We make the following assumption on the distribution of $a_{v}$ :

Assumption 1: $a_{v}$ is uniformly distributed in $[\alpha, \beta]$ (Fig. 1(b)) and this distribution is known to the operators.

We can then define two types of distributions:

Definition 3: The distribution of user type $a_{v}$ is called a narrow distribution if $\beta \leq 2 \alpha$ and a wide distribution if $\beta>2 \alpha$.

\section{MAin Results And CONTRibutions}

The model presented in the precedent section is evaluated in two scenarios: (1) a monopoly, in which a unique operator offers the wireless access, and (2) a duopoly, in which both operators compete for subscribers. In the rest of the paper, we obtain the pricing strategies that maximize the payoff of the operators in both settings.

We first show that the strategy maximizing the LBO revenue in a monopoly depends on the spread of the distribution of user types. For wide distribution of user type, the LBO maximizes its payoff by setting a high subscription fee such that only users with a high user type $a_{v}$ subscribe. In the case of a narrow distribution of user types, the LBO should set a subscription fee such that all users subscribe to its service. 
The payoff achieved with the wide distribution is higher than that achieved with the narrow distribution.

Next, we analyze the dynamics of the SCO in a monopoly; we consider two pricing strategies: static and dynamic pricing. We derive the equilibrium points of the SCO coverage with both pricing strategies and determine the price which achieves the maximal SCO payoff. We observe that the SCO payoff in monopoly is not only affected by the distribution of user types, but also by its initial provided coverage. We also observe that in the dynamic pricing strategy, the coverage $Q_{s}$ of the social community directly affects the subscription fee. We conclude that the SCO should first bootstrap its network with low prices to reach a fair coverage before adjusting its price to maximize its revenue. This conclusion nicely matches the behavior of real wireless social communities [3]. Finally, if the distribution of user types is narrow, then the SCO coverage at optimal point can converge to 1 whereas for a wide user type distribution, it is less than 1 . However, the achieved payoff is larger for the wide user type distribution.

We finally consider the co-existence of a LBO and a SCO and compute their respective best responses with a gametheoretic approach. The competition ends up in two scenarios depending again on the distribution of user types: (1) if $\beta \geq \frac{3}{2} \alpha$, then there is a Nash equilibrium in which both operators have subscribers, else (2) if $\beta<\frac{3}{2} \alpha$, then there is no Nash equilibrium for the game. A Nash equilibrium strategy profile results in lower subscription fees and more subscribers than monopoly scenario. We finally show that wireless operators do not have an economic incentive to deploy both a social community and a licensed band wireless access.

\section{REVENUE ANALYSIS OF A LICENSED BAND OPERATOR}

First, we assume that only the LBO provides wireless data access in the service area. We derive the final fraction of users $n_{\ell}$ who subscribes to the LBO. Given Assumption 1, one can easily obtain $n_{\ell}$ by,

$$
n_{\ell}=\frac{1}{\beta-\alpha}\left(\beta-\max \left\{\alpha, P_{\ell}\right\}\right)
$$

The LBO calculates its payoff by substituting (4) into (1):

$$
u_{\ell}=\frac{N}{\beta-\alpha}\left(\beta-\max \left\{\alpha, P_{\ell}\right\}\right) \cdot P_{\ell}-c_{\ell}
$$

The following lemma shows the optimal price of LBO.

Lemma 1: The optimal subscription fee that maximizes the LBO's payoff is:

$$
P_{\ell}^{o p t}=\max \left\{\alpha, \frac{\beta}{2}\right\}
$$

Proof: The proof is straightforward by taking the derivative of (5) with respect to $P_{\ell}$ and imposing it equal to 0 .

At this point, we emphasize by the following two Corollaries that the solutions for the optimal prices and payoffs depend on the distribution of user types defined by Definition 3 .

Corollary 1: Given a narrow distribution of user types, the optimal price of LBO is $P_{\ell}^{o p t}=\alpha$ and its corresponding payoff and fraction of subscribed users are $u_{\ell}^{o p t}=N \alpha-c_{\ell}$ and $n_{\ell}^{o p t}=1$, respectively.

Corollary 2: Given a wide distribution of user types, the optimal price of LBO is $P_{\ell}^{o p t}=\frac{\beta}{2}$ and the corresponding optimal payoff can be written as $u_{\ell}^{o p t}=\frac{N}{\beta-\alpha} \cdot \frac{\beta^{2}}{4}-c_{\ell}$. The maximum fraction of users $n_{\ell}^{o p t}$ who subscribe to LBO is $n_{\ell}^{o p t}=\frac{1}{2} \cdot \frac{\beta}{\beta-\alpha}$.

The above corollaries show that the maximum payoff depends on the distribution of user types $[\alpha, \beta]$. We observe that the optimal payoff of the LBO for a wide distribution of user types is always larger than that of narrow distribution. The calculated $n_{\ell}^{o p t}$ in Corollary 2 also shows that the LBO may ignore a subset of users (up to half of the users), in order to maximize its payoff.

\section{Dynamics of a Social COMmunity OPERATOR}

In this section, we assume that the SCO is the only wireless access provider and we study the evolution of its network. We assume that user $v$ will subscribe to the wireless access at time $t$ if and only if $u_{v}^{s}$ is strictly greater than zero for a given coverage $Q_{s}[t]$. Similar to the LBO we calculate the fraction of subscribed users as well as the achieved coverage of the SCO at time $t$ by,

$$
Q_{s}[t]=n_{s}[t]=\frac{1}{\beta-\alpha}\left(\beta-\max \left\{\alpha, \frac{P_{s}}{Q_{s}[t-1]}\right\}\right)
$$

The following two lemmas clarify the boundary conditions of Equation (7) for the number of subscribers and provided coverage. The proofs are straightforward considering Equation (7).

Lemma 2: For all $t>0$, if $Q_{s}[t-1]=0$ then $Q_{s}[t]=0$ and the SCO never forms.

Lemma 3: For all $t>0$, if $\frac{P_{s}}{Q_{s}[t-1]}<\alpha$ then $Q_{s}[t]=1$ and all users subscribe to SCO at time $t$.

Corollary 3: For all $t>0$, if $P_{s}=0$ then $Q_{s}[t+1]=$ $n_{s}[t+1]=1$.

If the condition of Lemma 3 does not hold, we denote the difference in term of coverage between two time steps $t$ and $t-1$, by $\Delta Q_{s}$ and express it as follow using Equation (7):

$$
\begin{aligned}
& \Delta Q_{s}=\quad Q_{s}[t]-Q_{s}[t-1] \\
& =\frac{-(\beta-\alpha) Q_{s}^{2}[t-1]+\beta \cdot Q_{s}[t-1]-P_{s}}{(\beta-\alpha) Q_{s}[t-1]},
\end{aligned}
$$

where positive and negative values of $\Delta Q_{s}$ express the improvement and degradation of the provided coverage of SCO at time $t$, respectively. We also define the equilibrium of SCO as follow:

Definition 4: For given values of $P_{s}, \alpha, \beta$, and $Q_{s}[t-1]$ the SCO is in equilibrium point $Q_{s}^{e q}$, if $\Delta Q_{s}=0$.

In the following analysis, we are interested in calculating of equilibrium points of the SCO, i.e., where the coverage and hence the fraction of subscribed users stabilizes. We are also interested in determining the type of convergence to the equilibrium points, i.e., decreasing or increasing. We will show 
that the convergence of the social community depends on the values of $P_{s}, \alpha, \beta$, and the initial coverage of SCO. Similar to $\mathrm{LBO}$, we obtain different solutions for various distributions of user types.

\section{A. Dynamics of SCO under Static Price}

In this section, we assume that the SCO applies a static pricing strategy (Definition 1). Assume for example that the coverage value $Q_{s}[t]$ is evaluated each month. It is reasonable to assume that the SCO keeps its price fixed for a longer time period to preserve the clarity of pricing for the users. We study the benefits of dynamic pricing strategies in Section V-C.

The following lemmas show the equilibrium points of SCO under static price strategy. The proofs of these lemmas are presented in Appendix A.

Lemma 4: For the narrow distribution of user types, there exist three equilibrium points: $Q_{s}^{e q}=\left\{0, Q_{s, 1}, 1\right\}$, where

$$
Q_{s, 1}=\frac{\beta-\sqrt{\beta^{2}-4(\beta-\alpha) P_{s}}}{2(\beta-\alpha)}
$$

Lemma 5: For the wide distribution of user types, there exist four equilibrium points: $Q_{s}^{e q}=\left\{0, Q_{s, 1}, Q_{s, 2}, 1\right\}$, where

$$
Q_{s, 2}=\frac{\beta+\sqrt{\beta^{2}-4(\beta-\alpha) P_{s}}}{2(\beta-\alpha)}
$$

The following lemmas show the type of convergence of SCO to the equilibrium points. The proofs are provided in Appendix A.

Lemma 6: Assume that the price of SCO is selected such that $P_{s} \leq \alpha$. For any distribution of user types, if $Q_{s}[t-1]<$ $Q_{s, 1}$ then $\lim _{t \rightarrow \infty} Q_{s}[t]=0$, otherwise $\lim _{t \rightarrow \infty} Q_{s}[t]=1$.

Lemma 7: For narrow distribution of user types, if $P_{s}>\alpha$ and for any given $Q_{s}[t-1]$ then $\lim _{t \rightarrow \infty} Q_{s}[t]=0$.

Fig. 2 and Fig. 3 (a) illustrate the dynamics of SCO for Lemma 6 and 7.

(a)

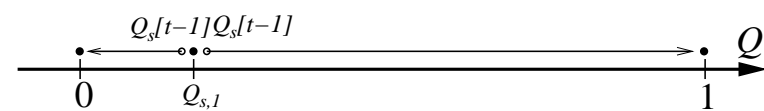

(b)

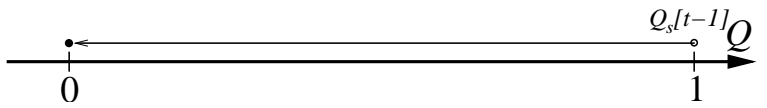

Fig. 2. Dynamics of the SCO for a narrow distribution of user types: (a) $0<P_{s} \leq \alpha$, (b) $P_{s}>\alpha$.

Lemma 8: For wide distribution of user types, if $\alpha<P_{s}<$ $\frac{\beta^{2}}{4(\beta-\alpha)}$ and $Q_{s}[t-1]<Q_{s, 1}$ then $\lim _{t \rightarrow \infty} Q_{s}[t]=0$. If $\alpha<$ $P_{s}<\frac{\beta^{2}}{4(\beta-\alpha)}$ and $Q_{s}[t-1]>Q_{s, 1}$ then $\lim _{t \rightarrow \infty} Q_{s}[t]=Q_{s, 2}$.

Lemma 9: For wide distribution of user types, if $P_{s}=$ $\frac{\beta^{2}}{4(\beta-\alpha)}$ and $Q_{s}[t-1]<Q_{s, 1}$ then $\lim _{t \rightarrow \infty} Q_{s}[t]=0$. For the same price if $Q_{s}[t-1]>Q_{s, 1}$ then $\lim _{t \rightarrow \infty} Q_{s}[t]=$ $Q_{s, 1}=Q_{s, 2}=\frac{\beta}{2(\beta-\alpha)}$.
Lemma 10: For wide distribution of user types, if $P_{s}>$ $\frac{\beta^{2}}{4(\beta-\alpha)}$, for any given $Q_{s}[t-1]$ then $\lim _{t \rightarrow \infty} Q_{s}[t]=0$.

Fig. 3 illustrates the dynamics of SCO for Lemma 8, 9, and 10. The following lemma shows the monotonous convergence of SCO coverage. The proof is in Appendix B.

Lemma 11: In Lemma 8 and 9, $Q_{s}$ converges monotonically to $Q_{s, 2}$.

(a)

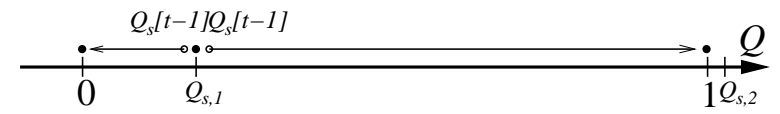

(b)

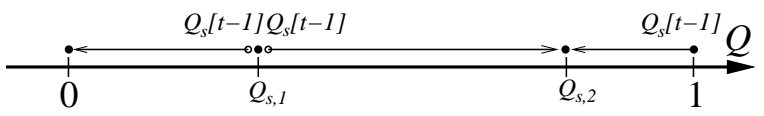

(c)

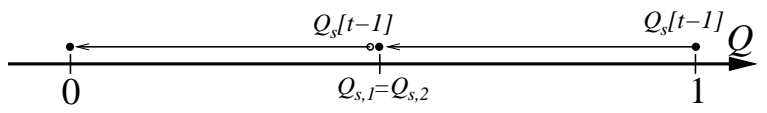

(d)

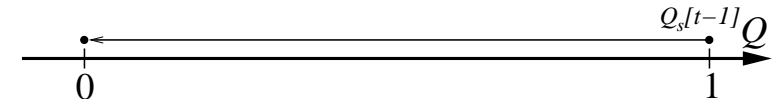

Fig. 3. Dynamics of SCO for a wide distribution of user types: (a) $0<$ $P_{s} \leq \alpha$, (b) $\alpha<P_{s}<\frac{\beta^{2}}{4(\beta-\alpha)}$, (c) $P_{s}=\frac{\beta^{2}}{4(\beta-\alpha)}$, (d) $P_{s}>\frac{\beta^{2}}{4(\beta-\alpha)}$.

Corollary 4: For any given $P_{s}>0, \alpha, \beta$, and time $t$ we observe that if the price selected by $\mathrm{SCO}$ is such that $Q_{s}[t-1]$ is less than $Q_{s, 1}$, then the SCO can never increase its coverage and consequently, the proportion of subscribers and its revenue. In this case $\lim _{t \rightarrow \infty} Q_{s}[t]=0$.

\section{B. Optimal Static Price}

In this subsection, we derive the optimal static price $P_{s}^{o p t}$ that maximizes the payoff of the SCO for various values of $Q_{s}[0], \alpha$ and $\beta$.

Theorem 1: For the narrow distribution of user types, the SCO should keep its price less than $\alpha$ to increase its coverage. For a given initial coverage $Q_{s}[0]$, the best value of $P_{s}$ which maximizes the SCO payoff is $P_{s}=Q_{s}[0] \cdot\left(\beta-(\beta-\alpha) \cdot Q_{s}[0]\right)$ and its corresponding maximum payoff is $N \cdot Q_{s}[0] \cdot(\beta-(\beta-$ $\left.\alpha) \cdot Q_{s}[0]\right)-c_{s}$.

Proof: As shown in Lemma 6 and 7, the SCO can increase its coverage if and only if $P_{s} \leq \alpha$. The upper limit of the convergence is then 1 (as illustrated in Fig. 2(a)). According to Corollary 4 and in order to increase the coverage, the SCO should select a price such that $Q_{s, 1}<Q_{s}[0]$. This means that:

$$
Q_{s, 1}=\frac{\beta-\sqrt{\beta^{2}-4(\beta-\alpha) P_{s}}}{2(\beta-\alpha)}=Q_{s}[0]-\epsilon
$$

where $\epsilon>0$ is a small positive value. From (11), we can express the value of $P_{s}$ as, $P_{s}=\left(Q_{s}[0]-\epsilon\right) \cdot(\beta-(\beta-\alpha)$. $\left.\left(Q_{s}[0]-\epsilon\right)\right)$, and for $\epsilon \rightarrow 0$,

$$
P_{s} \rightarrow Q_{s}[0] \cdot\left(\beta-(\beta-\alpha) \cdot Q_{s}[0]\right)
$$

The above price is always less than $\alpha$ for all $Q_{s}[0] \in$ $[0,1]$, hence it corresponds to convergence type presented in 
Lemma 6. The final fraction of subscribed users is $n_{s}=1$ and the corresponding payoff is:

$$
u_{s}=N \cdot n_{s} \cdot P_{s}-c_{s}=N \cdot Q_{s}[0] \cdot\left(\beta-(\beta-\alpha) \cdot Q_{s}[0]\right)-c_{s}
$$

Theorem 2: For the wide distribution of user types and if the initial coverage $Q_{s}[0]$ is smaller than $\frac{\alpha}{\beta-\alpha}$, the SCO should select a price $P_{s}$ such that $0<P_{s} \leq \alpha$. The best value of $P_{s}$ which maximizes the SCO payoff is then $P_{s}=Q_{s}[0] \cdot(\beta-$ $\left.(\beta-\alpha) \cdot Q_{s}[0]\right)$.

Proof: Having a closer look to the $Q_{s, 1}$ expression, we notice that for the value of $P_{s}$ in $[0, \alpha], Q_{s, 1}$ will be in $\left[0, \frac{\alpha}{\beta-\alpha}\right]$. For any $P_{s}$ bigger than $\alpha, Q_{s, 1}$ will be always greater than $\frac{\alpha}{\beta-\alpha}$. According to Corollary 4, the SCO should select a price such that $Q_{s}[0]>Q_{s, 1}$. Hence, since $Q_{s}[0]<\frac{\alpha}{\beta-\alpha}$ the price should be selected from $(0, \alpha]$ and the convergence type corresponds to Lemma 6 , similar to the narrow band distribution of user types. Then, the optimal static price and payoff function can be calculated by Equation (12) and (13).

Theorem 3: For the wide distribution of user types and if the initial coverage $Q_{s}[0]$ is bigger than $\frac{\alpha}{\beta-\alpha}$, the optimal static price is $P_{s}^{o p t}=\alpha$ for $2 \alpha<\beta \leq 3 \alpha$, otherwise the optimal price is $P_{s}^{o p t}=\frac{2}{9} \frac{\beta^{2}}{(\beta-\alpha)}$.

Proof: If $\frac{\alpha}{\beta-\alpha}<Q_{s}[0]<1$, the fraction of subscribed users can either converge to 1 (Lemma 6) or monotonically (Lemma 11) to $Q_{s, 2}$ (Lemma 8 and 9). We assume that the SCO selects a static price such that the scenario corresponds to the Lemma 8. In other words, the SCO selects a price in $\left(\alpha, \frac{\beta^{2}}{4(\beta-\alpha)}\right)$. Hence, the social community stabilizes in $Q_{s, 2}$ :

$$
\begin{aligned}
u_{s} & =\quad N \cdot P_{s} \cdot Q_{s, 2}-c_{s} \\
& =N \cdot P_{s} \cdot\left(\frac{\beta+\sqrt{\beta^{2}-4(\beta-\alpha) P_{s}}}{2(\beta-\alpha)}\right)-c_{s}
\end{aligned}
$$

We then calculate $P_{s}^{\text {opt }}$ that maximizes the SCO's payoff function $u_{s}$ by making the derivative of (14) with respect to $P_{s}$ and imposing it equal to zero.

$$
\frac{\partial u_{s}}{\partial P_{s}}=-\frac{N P_{s}}{\sqrt{\beta^{2}-4(\beta-\alpha) P_{s}}}+N \frac{\beta+\sqrt{\beta^{2}-4(\beta-\alpha) P_{s}}}{2(\beta-\alpha)}=0
$$

The optimal static price for this case is then:

$$
P_{s}^{o p t}=\frac{2}{9} \frac{\beta^{2}}{(\beta-\alpha)}
$$

For the price in (15), the following fraction of users subscribe to the SCO:

$$
n_{s}^{o p t}=Q_{s}=\frac{2}{3} \frac{\beta}{\beta-\alpha}
$$

Finally, we have to take into account that the SCO does not decrease his price below the lower bound of $\frac{P_{s}}{Q_{s}[t]}$, i.e.,

$$
\frac{P_{s}}{Q_{s}[t]}=\frac{P_{s}^{o p t}}{n_{s}^{o p t}}=\frac{\beta}{3}>\alpha
$$

This means that the above optimal static price exists if and only if $\beta>3 \alpha$. Accordingly, we can distinguish two subcases:
- If $2 \alpha<\beta \leq 3 \alpha$, then the optimal price in (15) is smaller than $\alpha$. One can show that the payoff function $u_{s}=$ $N \cdot P_{s} \cdot Q_{s, 2}-c_{s}$ is a concave function of $P_{s}$ and it is decreasing in the interval $\alpha<P_{s}<\frac{\beta^{2}}{4(\beta-\alpha)}$. This results in the optimal price $P_{s}^{o p t}=\alpha$ (i.e., $Q_{s, 2}=1$ ) and the maximum payoff value $u_{s}^{o p t}=N \alpha-c_{s}$.

- If $\beta>3 \alpha$, then the solution in (15) defines the optimal static price. Consequently, the fraction of subscribed users is defined in (16). For these $P_{s}^{o p t}$ and $n_{s}^{o p t}$ values, the optimal payoff of the SCO is $u_{s}^{o p t}=N \frac{4}{27} \frac{\beta^{3}}{(\beta-\alpha)^{2}}-c_{s}$.

We have seen that the initial coverage $Q_{s}[0]$, and the distribution of user types determine the range of optimal static prices from which the SCO can select its price. In the next subsection, we assume that the SCO applies dynamic pricing strategies to better adapt the price $P_{s}$ to the changing coverage.

\section{Dynamic Pricing}

Let us now assume that the $\mathrm{SCO}$ adjusts its price $P_{s}$ at time $t$ to follow the evolution of its network. The essential difference between static and dynamic pricing is that with dynamic pricing the SCO can maintain a lower price until a desired coverage is reached and then fine-tune the price. Since $\Delta Q_{s}$ must be strictly positive, we derive the following condition on the dynamic price:

$$
P_{s}[t]<-(\beta-\alpha) Q_{s}^{2}[t-1]+\beta Q_{s}[t-1]
$$

The right-hand side of (18) is always positive for all $Q_{s} \in$ $[0,1]$. Thus, the SCO maintains the increase of the coverage by selecting appropriate dynamic prices $P_{s}[t]$, such that,

$$
P_{s}[t]=-(\beta-\alpha) Q_{s}^{2}[t-1]+\beta Q_{s}[t-1]-\epsilon
$$

where $\epsilon$ is a small positive value. Similar to the static price strategy, two main scenarios can be distinguished.

1) Narrow Distribution of User Types: $P_{s}[t]$ is increasing in $[0,1]$ and its maximum value is $\alpha$ corresponding to $Q_{s}=1$. So the coverage of the SCO converges to 1 and $u_{s}=N \alpha-c_{s}$.

2) Wide Distribution of User Types: The maximum value of $P_{s}[t]$ is $\frac{\beta^{2}}{4(\beta-\alpha)}$ at $Q_{s}=\frac{\beta}{2(\beta-\alpha)}<1$. The SCO payoff is:

$$
u_{s}[t]=N \cdot \frac{1}{\beta-\alpha}\left(\beta-\frac{P_{s}[t]}{Q_{s}[t-1]}\right) \cdot P_{s}[t]-c_{s}
$$

Finally, we can express the SCO payoff as a function of $Q_{s}[t-$ 1] using Equation (19) when $\epsilon \rightarrow 0$ :

$u_{s}[t]=N(\beta-\alpha)\left(\beta-(\beta-\alpha) Q_{s}[t-1]\right) Q_{s}^{2}[t-1]-c_{s}$

Using Equation (21), we can obtain the best price and coverage that maximizes the SCO payoff, i.e., $Q_{s}^{o p t}=\frac{2}{3} \frac{\beta}{\beta-\alpha}$, $P_{s}^{o p t}=\frac{2}{9} \frac{\beta^{2}}{\beta-\alpha}$, and $u_{s}^{o p t}=\frac{4}{27} \frac{\beta^{3}}{(\beta-\alpha)^{2}}-c_{s}$. Considering the lower bound on $\frac{P_{s}}{Q_{s}[t]}$, we again conclude that the maximum value exists if $\beta>3 \alpha$. If $2 \alpha<\beta<3 \alpha$, the best price is $P_{s}^{o p t}=\alpha$ and coverage can potentially converge to 1 . 


\section{Coexistence of A SCO And A LBO}

So far, we evaluated the SCO and LBO individually and derived their optimal static and dynamic strategies in a monopoly. In this section, we consider a duopoly in which the simultaneous presence of the LBO and SCO can result in a competition for subscribers. We first show the possible outcomes of the duopoly. Then, we derive using a gametheoretic model [5], [6], [9] the best pricing strategy for each operator to maximize its payoff. We show that the existence of a Nash equilibrium depends on the distribution of user types.

\section{A. Interaction between $\mathrm{LBO}$ and $\mathrm{SCO}$}

We assume that the LBO provides full coverage service with price $P_{\ell}$ while the SCO offers service with coverage $Q_{s}$ for a given $P_{s}$. A user $v$ subscribes to the social community if its payoff with the $\mathrm{SCO}$ is positive and strictly greater than its payoff with the LBO, i.e., $u_{v}^{s}>u_{v}^{l}>0$. We express this inequality with respect to $a_{v}$ to exhibit the set of user $v$ that will prefer subscribing to the SCO, for a given $P_{\ell}, P_{s}$ and $Q_{s}$ :

$$
\begin{aligned}
a_{v} Q_{s}-P_{s} & >a_{v}-P_{\ell} \\
a_{v} & <\frac{P_{\ell}-P_{s}}{1-Q_{s}}
\end{aligned}
$$

Let us define $\theta=\frac{P_{\ell}-P_{s}}{1-Q_{s}}$. The lower bound on $a_{v}$ is obtained similarly:

$$
\begin{aligned}
a_{v} Q_{s}-P_{s} & >0 \\
a_{v} & >\frac{P_{s}}{Q_{s}}
\end{aligned}
$$

Considering (22) and (23), three possible scenarios can be distinguished by the following lemmas.

Lemma 12: For given values of $P_{s}, Q_{s}$, and $P_{\ell}$, if $P_{\ell} \in$ $\left(0, \frac{P_{s}}{Q_{s}}\right]$, then $\theta \leq \frac{P_{s}}{Q_{s}}$ and all mobile users subscribe to the LBO.

Lemma 13: For given values of $P_{s}, Q_{s}$, and $P_{\ell}$, if $P_{\ell} \in$ $\left(\frac{P_{s}}{Q_{s}}, \beta\left(1-Q_{s}\right)+P_{s}\right)$, then $\frac{P_{s}}{Q_{s}}<\theta<\beta$ and a user with $a_{v} \in$ $\left(\frac{P_{s}}{Q_{s}}, \theta\right)$ subscribes to the SCO whereas a user with $a_{v} \in[\theta, \beta]$ subscribes to the LBO.

Lemma 14: For given values of $P_{s}, Q_{s}$, and $P_{\ell}$, if $P_{\ell} \in$ $\left[\beta\left(1-Q_{s}\right)+P_{s}, \infty\right)$, then $\theta \geq \beta$ and all LBO mobile users subscribe to the SCO.

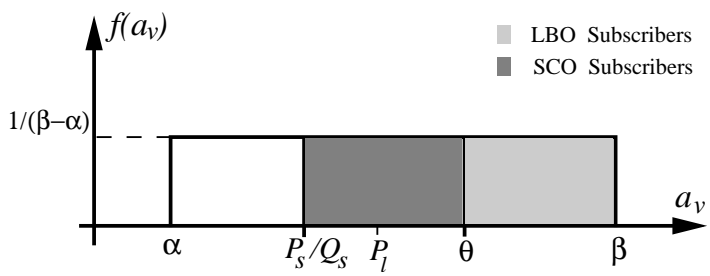

Fig. 4. Uniform distribution of user types and a scenario in which both operators have some subscribers (Lemma 13).

The above lemmas show all possible outcomes of the coexistence of two operators. Fig. 4 illustrates the interaction of operators under the conditions of Lemma 13. In the next section, we model and evaluate the strategies of the operators using a game-theoretic approach.

\section{B. Game Model}

We define a two-player non-cooperative pricing game $G$ with the operators as players. The strategy of operator $\sigma_{i}=P_{i}$, $i \in\{\ell, s\}$ determines its subscription fee. We call the set of strategies of all players a strategy profile $\sigma=\left\{\sigma_{1}, \sigma_{2}\right\}=$ $\left\{P_{\ell}, P_{s}\right\}$. The players share the same strategy set $\Sigma=[0, \infty)$. Note that for a given strategy profile $\sigma$, one of the three scenarios, which are described with Lemma 12, 13, and 14 may take place. The $Q_{s}$ will be then equal to 0 or 1 , or can be calculated by solving the following equation:

$$
Q_{s}=\frac{1}{\beta-\alpha}\left(\theta-\frac{P_{s}}{Q_{s}}\right)
$$

In order to get an insight into the strategic behavior of the operators, we apply the following game-theoretic concepts. First, let us introduce the concept of best response. We can write $b r_{i}\left(\sigma_{j}\right)$, the best response of player $i$ to the opponent's strategy $\sigma_{j}$ as follows.

Definition 5: The best response of player $i$ to the profile of strategies $\sigma_{j}$ is a strategy $\sigma_{i}$ such that:

$$
b r_{i}\left(\sigma_{j}\right)=\arg \max _{\sigma_{i} \in \Sigma} u_{i}\left(\sigma_{i}, \sigma_{j}\right)
$$

If two strategies are mutual best responses to each other, then no player has a motivation to deviate from the given strategy profile. To identify such strategy profiles in general, Nash introduced the concept of Nash equilibrium [8]:

Definition 6: The pure-strategy profile $\sigma^{*}$ constitutes a Nash equilibrium if, for each player $i$,

$$
u_{i}\left(\sigma_{i}^{*}, \sigma_{j}^{*}\right) \geq u_{i}\left(\sigma_{i}, \sigma_{j}^{*}\right), \forall \sigma_{i} \in \Sigma
$$

where $\sigma_{i}^{*}$ and $\sigma_{j}^{*}$ are the Nash equilibrium strategies of player $i$ and $j$, respectively.

In other words, in a Nash equilibrium, none of the players can unilaterally change his strategy to increase his utility. In the next section, we derive the best pricing strategies for both operators.

\section{LBO and SCO Pricing Strategy}

When the two operators are competing for subscribers, the fraction of users who stay with the LBO for a given $P_{s}$ and $Q_{s}$ is:

$$
n_{\ell}=\left\{\begin{array}{llr}
\frac{1}{\beta-\alpha}\left(\beta-P_{\ell}\right) & \text { if } & \theta<\frac{P_{s}}{Q_{s}} \\
\frac{1}{\beta-\alpha}(\beta-\theta) & \text { if } & \frac{P_{s}}{Q_{s}}<\theta<\beta \\
0 & \text { if } & \theta>\beta
\end{array}\right.
$$

Similarly the fraction of users in the SCO is:

$$
n_{s}=Q_{s}=\left\{\begin{array}{llr}
0 & \text { if } & \theta<\frac{P_{s}}{Q_{s}} \\
\frac{1}{\beta-\alpha}\left(\theta-\frac{P_{s}}{Q_{s}}\right) & \text { if } & \frac{P_{s}}{Q_{s}}<\theta<\beta \\
\frac{1}{\beta-\alpha}\left(\beta-\frac{P_{s}}{Q_{s}}\right) & \text { if } & \theta>\beta
\end{array}\right.
$$

If $\theta<\frac{P_{s}}{Q_{s}}$, then the best response of the LBO can be calculated in the same way as in the monopoly scenario (i.e., $P_{\ell}^{o p t}$ in Section IV), whereas the SCO's payoff is zero. If $\theta>\beta$ the best response of the SCO can be calculated as presented in 
Section V, whereas the LBO's payoff is zero. The following theorem shows the best response of the operators for the given values of $P_{s}, P_{\ell}$, and $Q_{s}$, when $\frac{P_{s}}{Q_{s}}<\theta<\beta$.

Theorem 4: If $\frac{P_{s}}{Q_{s}}<\theta<\beta$, the LBO's and SCO's best responses are $b r_{\ell}\left(P_{s}\right)=\frac{\beta\left(1-Q_{s}\right)+P_{s}}{2}$ and $b r_{s}\left(P_{\ell}\right)=\frac{P_{\ell} Q_{s}}{2}$ respectively.

Proof: If $\frac{P_{s}}{Q_{s}}<\theta<\beta$, the LBO's payoff can be calculated by:

$$
u_{\ell}=\frac{N}{\beta-\alpha}(\beta-\theta) P_{\ell}-c_{\ell}
$$

Maximizing (29) with respect to $P_{\ell}$ results in:

$$
\frac{\partial u_{\ell}}{\partial P_{\ell}}=N \frac{\beta\left(1-Q_{s}\right)-2 P_{\ell}+P_{s}}{(\beta-\alpha)\left(1-Q_{s}\right)}=0
$$

We obtain the LBO's best response:

$$
b r_{\ell}\left(P_{s}\right)=\frac{\beta\left(1-Q_{s}\right)+P_{s}}{2}
$$

Similarly, the SCO's payoff can be calculated by:

$$
u_{s}=\frac{N}{\beta-\alpha}\left(\theta-\frac{P_{s}}{Q_{s}}\right) P_{s}-c_{s}
$$

Maximizing (31) with respect to $P_{s}$ :

$$
\frac{\partial u_{s}}{\partial P_{s}}=N \frac{P_{\ell}-\frac{2 P_{s}}{Q_{s}}}{(\beta-\alpha)\left(1-Q_{s}\right)}=0
$$

We obtain the SCO's best response:

$$
b r_{s}\left(P_{\ell}\right)=\frac{P_{\ell} Q_{s}}{2}
$$

We observe that $b r_{\ell}\left(P_{s}\right)$ depends on the strategy of the social community and its coverage. Similarly, $b r_{s}\left(P_{\ell}\right)$ depends on the subscription fee of the LBO and on the offered coverage by the SCO. Finally, the LBO's and SCO's payoff at the best response are $u_{\ell}\left(b r_{\ell}\left(P_{s}\right)\right)=N \frac{\left(\beta\left(1-Q_{s}\right)+P_{s}\right)^{2}}{4(\beta-\alpha)\left(1-Q_{s}\right)}-c_{\ell}$ and $u_{s}\left(b r_{s}\left(P_{\ell}\right)\right)=$ $N_{4(\beta-\alpha)\left(1-Q_{s}\right)}-c_{s}$ respectively. It is worth mentioning that the calculated payoff at the best response strategies could be negative depending on the infrastructure $\operatorname{cost} c_{s}$ and $c_{\ell}$.

\section{Existence of a Nash Equilibrium}

When both operators use their best response strategies, the system may converge to a Nash equilibrium, if it exists. The following theorem gives the sufficient conditions for the existence of the Nash equilibrium and its value.

Theorem 5: Game $G$ has a Nash equilibrium if the distribution of user types is such that $\beta \geq \frac{3}{2} \alpha$. The Nash equilibrium strategy profile is then $\left(P_{\ell}^{*}, P_{s}^{*}\right)=\left(\frac{\beta}{2} \cdot \frac{1-Q_{s}^{*}}{1-\frac{Q_{s}^{*}}{4}}, \frac{\beta Q_{s}^{*}}{4} \cdot \frac{1-Q_{s}^{*}}{1-\frac{Q_{s}^{*}}{4}}\right)$, where $Q_{s}^{*}=2-\sqrt{4-\frac{\beta}{\beta-\alpha}}$. If the distribution of user types is such that $\beta<\frac{3}{2} \alpha$, there is no Nash equilibrium.
Proof: The Nash equilibrium strategy profile can be computed using the best response of both operators defined in (30) and (32), i.e.,

$$
\begin{aligned}
& P_{\ell}^{*}=b r_{\ell}\left(b r_{s}\left(P_{\ell}\right)\right)=\frac{\beta}{2} \cdot \frac{1-Q_{s}}{1-\frac{Q_{s}}{4}} \\
& P_{s}^{*}=b r_{s}\left(b r_{\ell}\left(P_{s}\right)\right)=\frac{\beta Q_{s}}{4} \cdot \frac{1-Q_{s}}{1-\frac{Q_{s}}{4}}
\end{aligned}
$$

Using (28) for the given $\left(P_{\ell}^{*}, P_{s}^{*}\right)$, the coverage of the SCO at Nash equilibrium point can be computed by solving the following quadratic expression:

$$
-\frac{(\beta-\alpha)}{4} Q_{s}^{* 2}+(\beta-\alpha) Q_{s}^{*}-\frac{\beta}{4}=0
$$

Equation (35) has two solutions: $Q_{s, 1,2}^{*}=2 \pm \sqrt{4-\frac{\beta}{\beta-\alpha}}$. Because $Q_{s}^{*}$ must belong to the interval $[0,1]$, the only acceptable solution is $Q_{s, 2}^{*}=2-\sqrt{4-\frac{\beta}{\beta-\alpha}}$. The Nash equilibrium profile exists (i.e., $Q_{s, 2}^{*}$ is less than 1 ) if the distribution of user types is such that $\beta \geq \frac{3}{2} \alpha$. If $\beta<\frac{3}{2} \alpha$ there is no SCO coverage, $Q_{s}$, which satisfies (35) for the calculated Nash equilibrium $\left(P_{\ell}^{*}, P_{s}^{*}\right)$.

We do not evaluate the time needed to converge to the Nash equilibrium, but it depends on the original pricing strategies and original offered coverage by the SCO.

As a numerical example, if $\alpha=0$ then $Q_{s, 2}^{*}=2-\sqrt{3}$. In other words, the coverage achieved by the social community at the Nash equilibrium is about $27 \%$. This coverage is much lower than that achieved in the case of a monopoly (Equation (16)), i.e., $Q_{s}^{o p t}=66 \%$. The SCO and LBO strategies are $P_{\ell}^{*}=2 \beta \frac{-1+\sqrt{3}}{2+\sqrt{3}} \cong 0.39 \beta$ and $P_{s}^{*}=\beta \frac{-5+3 \sqrt{3}}{2+\sqrt{3}} \cong 0.05 \beta$. The LBO price decreases from $0.5 \beta$ (Equation (6)) to $0.39 \beta$. Similarly, the SCO price at equilibrium point (i.e., $0.05 \beta$ ) is much lower than that of the monopoly (Equation (15)), i.e., $0.22 \beta$. However, the total number of users considering both LBO and SCO subscribers is now equal to $87 \%$. In summary, as a consequence of competition, prices are lowered and more users are served. This means that operators provide service to more users with cheaper price at Nash equilibrium point.

\section{RELATED WORK}

The wireless community networks over unlicensed band have been recently deployed by some ISPs such as Free [4] in France or FON, a worldwide WiFi community operator funded by Google and Skype [3]. A charging model for wireless social community networks without a centralized authority is proposed by Efstathiou et al. [1]. Their solution relies on reciprocity among subscribers. In [10], Zemlianov and de Veciana evaluate using a stochastic geometric model, the cooperation between licensed band WAN and WLAN service providers. Using different classes of payoff functions, they focus on the mobile user decision and show that the class of payoff functions that are congestion dependent provide on average a much better performance to users than the simple proximity-based decision strategy. 
To determine the price to charge for a product is an old problem. In the neoclassical period, the models assumed perfect information about the market demand for a product, i.e., the profit function was deterministic. We take this approach in our paper for simplicity. Our pricing results in monopoly fit in the pricing literature. The duopoly scenario with linear payoff functions has also been studied extensively. To the best of our knowledge, we are the first to apply it to the study of the coexistence of SCOs with traditional LBOs.

\section{CONCLUSIONS}

In this paper, we study how the evolution of social community networks is conditioned by the initial wireless coverage, the subscription price and user preferences for coverage. In a monopoly, our model determines the optimal pricing strategy for the social community. It shows that without a minimal initial coverage, a social community cannot gain momentum. Thus, a SCO should select a low price at first to reach a fair coverage while working at loss (i.e., $u_{s}<0$ ). This strategy nicely matches the behavior of wireless social communities. The wide distribution of users types is also more profitable as it generates more revenue that a narrow distribution of user types. Our model helps wireless social communities to determine when the initial coverage is sufficient, and which price to set then. The model also provides pricing strategies for both type of user preferences.

Subsequently, we put the SCO and LBO in competition. We identify the scenarios in which the SCO or LBO can eliminate each others. We then derive scenarios where both social communities and traditional operators coexist. We find that for a sufficient distribution of user types $\beta \leq \frac{3}{2} \alpha$, a unique Nash Equilibrium exists. We also give the prices that the operators should use to reach the NE. The model indicates to operators in which market segment they can coexists.

However, the model suffers from stringent assumptions such as linear payoff functions, linear dependence between the coverage and number of users, and user preferences distributions. If its results should not be applied as is in practice, they still provide cues about the SCOs potential as a wireless operator. In the future, we intent to extend our model by considering more realistic assumptions and observe how they affect the achieved results.

\section{Open Questions And Future Work}

The model presented in this paper can be extended to consider various issues:

- Multiple Operators: In this paper, we assume that only one LBO and SCO provide wireless access. In reality, many centralized networks exist and we predict that concurrent social community networks are going to emerge. The presence of several social communities might decrease their competitiveness with respect to LBOs. Furthermore, we assume that the ISPs providing wired access allow the social communities to share this service among the users. The competition or coalition between the ISPs and SCOs could influence the outcome of the study [4]. For example, an ISP can cooperate with a SCO to provide a full coverage solution comparable to that of a LBO.

- Distribution of User Types: We have also seen that the solution depends on the distribution of the user types. We assume that this distribution is uniform and known to the operators. If this distribution is not known to the operators, then they have to base their decisions on incomplete information obtained from past behavior of users. The coexistence of the two operators with incomplete information can be studied in a Bayesian game. We also have to mention that we assume a continuous distribution of user types in the interval $\{\alpha, \beta\}$. This enables the social community to maintain a continuous growth. If the distribution of user types is not continuous, then the growth of the social community might be interrupted.

- User Preferences and QoS of the Social Community: We assume that the users choose their operator solely based on the provided coverage. In addition, we assume that the coverage grows linearly with the number of users (as shown in Fig. 1). It is reasonable to assume that the users also take other aspects into account (e.g., throughput and delay) when choosing their operator. Furthermore, the coverage of the social community depends on the location of the APs deployed by the users. Due to the lack of network planning, this coverage might be uneven, irrespectively of the high number of subscribers. The QoS is also affected by the density of APs; interference due to high density of APs can reduce the efficiency of the social community network. We extended our model for QoS functions that include the offered throughput by the operators and the interference generated by the AP of other users. Unfortunately, we cannot present our preliminary results in this paper due to the lack of space.

- Costs: We can further extend our model by considering the switching cost for users. Typically, users have to suffer a penalty if they want to switch between operators. This cost makes the switching more difficult and might result in a lock-in effect, where some users stay with their current operator, although they could enjoy a better service with another operator. Furthermore, we assume that the operators have a fixed network infrastructure cost. This might be the case for the LBO who has to maintain the same infrastructure independently of the number of its subscribers. The cost of the SCO, however, might depend on the number of subscribers since it has to provide the APs for new users.

\section{REFERENCES}

[1] E. C. Efstathiou, P. A. Frangoudis, and G. C. Polyzos. Stimulating participation in wireless community networks. In INFOCOM, 2006.

[2] N. Feamster, L. Gao, and J. Rexford. How to lease the internet in your spare time. In ACM SIGCOMM CCR, 2007.

[3] FON. http://www.fon.com/.

[4] Free. Internet service provider. http://www.free.fr/.

[5] D. Fudenberg and J. Tirole. Game Theory. MIT Press, 1991.

[6] R. Gibbons. A Primer in Game Theory. Prentice Hall, 1992.

[7] M. M. Halldorsson, J.Y. Halpern, L. E. Li, and V.S. Mirrokni. On spectrum sharing games. In ACM symposium PODC, USA, 2004.

[8] J. Nash. Non-cooperative games. The Annals of Mathematics, 54(2):286-295, 1951.

[9] M. J. Osborne and A. Rubinstein. A Course in Game Theory. The MIT Press, Cambridge, MA, 1994. 
[10] A. Zemlianov and G. de Veciana. Cooperation and decision making in wireless multiprovider setting. In INFOCOM, 2005.

\section{APPENDIX A}

PROOF OF LEMMAS 4 TO 10

Given that $\beta>\alpha$, the denominator of (8) is always positive, hence we focus on the numerator. Let's assume that $E=$ $-(\beta-\alpha) Q_{s}^{2}[t-1]+\beta \cdot Q_{s}[t-1]-P_{s}$.

The roots of the numerator $E$ (i.e., $\Delta Q_{s}=0$ ) are the equilibrium points of $\mathrm{SCO}$ as defined in Definition 4. Note that $E$ is a quadratic form equation which has maximum two roots and one global maximum point. We call these roots, $Q_{s, 1}$ and $Q_{s, 2}$, and they can be written by following expression:

$$
Q_{s, 1,2}=\frac{\beta \pm \sqrt{\beta^{2}-4(\beta-\alpha) P_{s}}}{2(\beta-\alpha)}
$$

We are interested in determining these roots (i.e., if they occur in $[0,1])$ for values of $\alpha, \beta$, and $P_{s}$. Let us first study the behavior of $E$ by taking its derivation and calculating its global maximum point and the corresponding coverage, i.e.,

$$
\frac{\partial E}{\partial Q_{s}}=-2(\beta-\alpha) \bar{Q}_{s}+\beta=0
$$

So $E$ has a global maximum point equal to $\frac{\beta^{2}}{4(\beta-\alpha)}-P_{s}$ at

$$
\bar{Q}_{s}=\frac{\beta}{2(\beta-\alpha)}
$$

Note that, $\bar{Q}_{s}$ is less than 1 for $\beta>2 \alpha$ and it is bigger or equal to 1 for $\beta \leq 2 \alpha$. Considering this we provide the proofs of Lemmas for narrow and wide distribution of user types in the following parts.

If the distribution of user types is such that $\beta \leq 2 \alpha, \frac{\partial E}{\partial Q_{s}}$ is always positive for all $Q_{s} \in[0,1]$ and $E$ is always increasing in this interval. Note that the values of $E$ at 0 and 1 are $-P_{s}$ and $\alpha-P_{s}$ respectively. Hence, if $\alpha-P_{s}>0$, the first root (i.e., $\left.Q_{s, 1}\right)$ is in $[0,1]$ and the second one (i.e., $Q_{s, 2}$ ) is greater than one. On the other hand, if $\alpha-P_{s}<0$, then there is no root in $[0,1]$ and $\Delta Q_{s}$ is always negative (i.e., proof of Lemma 4).

Having the above calculation, we can now distinguish two scenarios for the convergence of the social community coverage depending on the values of $P_{s}, \alpha, \beta$, and initial coverage, $Q_{s}[t-1]$, when $\beta \leq 2 \alpha$ as presented in Fig. 2 .

(a) If $0<P_{s} \leq \alpha$, then $0<Q_{s, 1}<1$ and $Q_{s, 2}>1$. Two subcases can be distinguished as follows (i.e., proof of Lemma 6):

$$
\begin{aligned}
& \text { - If } Q_{s}[t-1]<Q_{s, 1} \text {, then } \Delta Q_{s}<0 \text { and } \\
& \quad \lim _{t \rightarrow \infty} Q_{s}[t]=0 . \\
& \text { - If } Q_{s}[t-1]>Q_{s, 1} \text {, then } \Delta Q_{s}>0 \text { and } \\
& \quad \lim _{t \rightarrow \infty} Q_{s}[t]=1 .
\end{aligned}
$$

(b) If $P_{s}>\alpha$, then there is no convergence point in $[0,1], \Delta Q_{s}<0$, and $\lim _{t \rightarrow \infty} Q_{s}[t]=0$ (i.e., proof of Lemma 7).

For a wide distribution of user types, we conclude that $0<$ $\bar{Q}_{s}<1$. This means that the global maximum point of $E$ occurs in $[0,1]$. Hence, the SCO can have four equilibrium points in $[0,1]$ (i.e., proof of Lemma 5). Note that the values of $E$ at 0,1 , and the global maximum point are $-P_{s}, \alpha-P_{s}$, and $\frac{\beta^{2}}{4(\beta-\alpha)}-P_{s}$, respectively and $\frac{\beta^{2}}{4(\beta-\alpha)}>\alpha$. Similar to the previous case, considering the sign of $\alpha-P_{s}$ and $\frac{\beta^{2}}{4(\beta-\alpha)}-P_{s}$, we can now distinguish four scenarios for the convergence of the social community coverage as presented in Fig. 3.

(a) If $0<P_{s} \leq \alpha$, then $0<Q_{s, 1}<1$ and $Q_{s, 2} \geq 1$. It is worth mentioning that since $Q_{s, 1}=\frac{\beta-\sqrt{\beta^{2}-4(\beta-\alpha) P_{s}}}{2(\beta-\alpha)}$ and $\beta>2 \alpha$ then $Q_{s, 1} \in\left[0, \frac{\alpha}{\beta-\alpha}\right]$. We can thus distinguish these subcases (i.e., proof of Lemma 6):

- If $Q_{s}[t-1]<Q_{s, 1}$, then $\Delta Q_{s}$ is negative and $\lim _{t \rightarrow \infty} Q_{s}[t]=0$.

- If $Q_{s}[t-1]=Q_{s, 1}$ then $Q_{s}[t]=Q_{s, 1}$ for any $t$.

- If $Q_{s}[t-1]>Q_{s, 1}$, then $\Delta Q_{s}$ is positive and $\lim _{t \rightarrow \infty} Q_{s}[t]=1$.

(b) If $\alpha<P_{s}<\frac{\beta^{2}}{4(\beta-\alpha)}$, then $0<Q_{s, 1}<Q_{s, 2} \leq 1$ and the convergence dynamics depends again on $Q_{s}[t-1]$ (i.e., proof of Lemma 8).

- If $Q_{s}[t-1]<Q_{s, 1}$, then $\Delta Q_{s}<0$ and $\lim _{t \rightarrow \infty} Q_{s}[t]=0$.

- If $Q_{s}[t-1]=Q_{s, 1}$ then $Q_{s}[t]=Q_{s, 1}$ for any $t$.

- If $Q_{s, 1}<Q_{s}[t-1]<Q_{s, 2}$, then $\Delta Q_{s}>0$ and $\lim _{t \rightarrow \infty} Q_{s}[t]=Q_{s, 2}$.

- If $Q_{s}[t-1]=Q_{s, 2}$ then $\Delta Q_{s}=0$ and $Q_{s}[t]=Q_{s, 2}$ for any $t$.

- If $Q_{s}[t-1]>Q_{s, 2}$ then $\lim _{t \rightarrow \infty} Q_{s}[t]=Q_{s, 2}$.

(c) If $P_{s}=\frac{\beta^{2}}{4(\beta-\alpha)}$ then $\Delta Q_{s} \leq 0$ and $Q_{s}[t]$ is always nonincreasing. Furthermore, $Q_{s, 2}=Q_{s, 1}=\frac{\beta}{2(\beta-\alpha)}<1$. In summary, these subcases exist (i.e., proof of Lemma 9):

- If $Q_{s}[t-1]<Q_{s, 1}=Q_{s, 2}$ then $\Delta Q_{s}<0$ and $\lim _{t \rightarrow \infty} Q_{s}[t]=0$.

- If $Q_{s}[t-1]=Q_{s, 1}=Q_{s, 2}$ then $\Delta Q_{s}=0$ and $Q_{s}[t]=Q_{s, 1}=Q_{s, 2}=\frac{\beta}{2(\beta-\alpha)}$ for any $t$.

- If $Q_{s}[t-1]>Q_{s, 1}=Q_{s, 2}$ then $\lim _{t \rightarrow \infty} Q_{s}[t]=$ $Q_{s, 1}=Q_{s, 2}=\frac{\beta}{2(\beta-\alpha)}$.

(d) Finally, if $P_{s}>\frac{\beta^{2}}{4(\beta-\alpha)}$, then $Q_{s, 1}$ and $Q_{s, 2}$ do not exist, $\Delta Q_{s}$ is always negative and thus $\lim _{t \rightarrow \infty} Q_{s}[t]=0$ for all $Q_{s}[t-1]$ (i.e., proof of Lemma 10).

\section{APPENDIX B}

\section{PROOF OF LEMMA 11}

We consider the case (b) in Fig. 3, where $\frac{\alpha}{\beta-\alpha}<Q_{s}[0]<1$ and $P_{s}$ is selected such that $Q_{s}$ increases and converges to $Q_{s, 2}$. Following we prove that $Q_{s}$ will never take a value bigger than $Q_{s, 2}$ during the convergence process. If $P_{s}$ is selected such that for a given $Q_{s}[t-1], Q_{s}[t]>Q_{s}[t-1]$ and $Q_{s}[t]>Q_{s, 2}$ then we can write:

$$
\begin{aligned}
\frac{1}{\beta-\alpha}\left(\beta-\frac{P_{s}}{Q_{s}[t-1]}\right) & >\frac{\beta+\sqrt{\beta^{2}+4(\beta-\alpha) P_{s}}}{2(\beta-\alpha)} \\
P_{s} & >\beta Q_{s}[t-1]-(\beta-\alpha) Q_{s}^{2}[t-1]
\end{aligned}
$$


This means that if $Q_{s}[t]>Q_{s, 2}, P s$ should be greater than $\beta Q_{s}[t-1]-(\beta-\alpha) Q_{s}^{2}[t-1]$. Let us assume that $P_{s}=\beta Q_{s}[t-1]-(\beta-\alpha) Q_{s}^{2}[t-1]+\epsilon$, where $\epsilon$ is a small positive number. We can calculate $\Delta Q_{s}$ using Equation (8), i.e., $\Delta Q_{s}=\frac{-\epsilon}{(\beta-\alpha) Q_{s}[t-1]}$ which is always negative. Thus $Q_{s}[t]$ could not be greater than $Q_{s, 2}$ if $Q_{s}[t]$ is increasing. Similar proofs for convergence from right side as well as monotonous convergence to $Q_{s, 1}$ in Lemma 9 can be presented. 